\title{
Aprendizaje de Secuencias de Acción Para la Toma de Decisiones en Instalaciones DomóTicas
}

\author{
Vicente Botón-Fe rnánde $z^{1, *}$, José Luis Redondo-García, Adolfo Lozano-Tello
}

Quercus Software Engineering Group Universidad de Extremadura Escuela Politécnica, Campus Universitario s/n, 10071

\begin{abstract}
Conocer los hábitos de comportamiento de los individuos puede contribuir a la to ma de decisiones en entornos centrados en los humanos. Este trabajo presenta un modelo de aprendizaje dentro del proyecto IntelliDo mo, un sistema capaz de aprender los hábitos de los individuos y de realizar la toma de decisiones para generar automáticamente reglas de producción que anticipen las actividades periódicas y frecuentes de los usuarios. La capa de aprendizaje incorpora nuevas características como la detección de secuencias de acción, ya que los hábitos pueden definirse mejor si están relacionados con acciones encadenadas, creando relaciones de acción-acción.
\end{abstract}

Keywords Inteligencia A mbiental, To ma de Decisiones, Algoritmos de Aprendizaje, OntologÍAs, Intellido mo

\section{Introducción}

En los últimos años, el diseño de entornos inteligentes se ha convertido en una de las áreas de investigación que está ganando importancia rápidamente en campos como la asistencia sanitaria, la eficiencia energética, etc donde la interacción entre los usuarios y el entorno constituye un factor fundamental. Además, factores como el envejecimiento de la población, el coste de los servicios de salud, y la importancia de que las personas vivan con independencia en sus hogares, están acrecentando aún más la necesidad de desarrollo de estas tecnologías. Una característica importante que deben poseer estos entornos es la habilidad para adaptarse a las preferencias de los habitantes y tener la versatilidad para tomar decisiones en todo tipo de situaciones. En este sentido, encontrar patrones de comportamiento dentro de una secuencia de eventos para predecir acciones futuras puede encaminarnos hacia la consecución de dichos objetivos. En consecuencia, el sistema será capaz de identificar y reconocer el comportamiento humano para utilizar este conocimiento en la to ma de decisiones, anticipándose a las necesidades y preferencias de los habitantes. Por otra parte, la información adquirida por estas técnicas de detección de comportamiento podría utilizarse para llevar un seguimiento de las actividades periódicas de los usuarios y el orden en que las realizan, lo cual es especialmente interesante en el caso de personas ancianas para detectar posibles casos de Alzheimer y actuar en consecuencia[1].

* Corresponding author:

vboton@unex.es (Vicente Botón-Fernández )

Published online at http://journal.sapub.org/computer

Copyright (C) 2012 Scientific \& Academic Publishing. All Rights Reserved
Obviamente, para descubrir estos hábitos y preferencias se necesita una tarea previa de aprendizaje. En un entorno inteligente, aprendizaje significa que el entorno tiene que adquirir conocimiento sobre las preferencias de los usuarios, su comportamiento común o patrón de actividad de una forma discreta y transparente para el usuario.

El uso de ontologías[2] para clasificar los tipos de dispositivos domóticos y su funcionalidad supone una forma apropiada de representación de los conceptos de estos entornos. Las ontologías y las reglas SW RL (Semantic Web Rule Language) proporcionan una defin ición precisa de una taxonomía domótica: la localización de los dispositivos físicos, sus características y categorías funcionales (seguridad, eficiencia energética, confort...), y las relaciones entre ellos. Además, estas representaciones son reutilizables, por lo que otros usuarios pueden utilizarlas para clasificar sus propios componentes domóticos y construir reglas que permitan controlar el co mportamiento del entorno.

Este artículo describe las nuevas funcionalidades de la capa de aprendizaje de IntelliDomo, una propuesta de minería de datos para la gestión de sistemas AmI (Ambient Intelligence) y para el aprendizaje de comportamiento humano basada en ontologías, incluyendo ahora el reconocimiento de secuencias de acción. El resto del artículo se estructura de la siguiente forma: la sección 2 presenta distintas técnicas que se han utilizado hasta ahora para el desarrollo de modelos de aprendizaje en entornos inteligentes. La sección 3 describe la arquitectura y las características principales de los distintos módulos que conforman nuestro modelo de aprendizaje. La sección 4 profundiza en el funcionamiento de los algoritmos utilizados para el desarrollo del módulo de aprendizaje; y en la sección 5 se presenta uno de los casos de estudio utilizados para probar la validez de la aplicación. Por último, la sección 6 
está dedicada a las conclusiones y trabajos futuros.

\section{Minería de Datos Aplicada a Entornos Inteligentes}

En la actualidad, el desarrollo de técnicas de minería de datos para procesar e interpretar los datos capturados de un conjunto de sensores y actuadores en un entorno inteligente está ganando importancia debido a la necesidad de satisfacer los gustos de los usuarios y anticiparse a sus hábitos frecuentes. Existe un número significativo de proyectos de investigación sobre sistemas AmI que controlan y automatizan las tareas de los usuarios con distinto grado de éxito, y la mayoría de estos trabajos se centran en unos aspectos my concretos debido a la alta complejidad de estas arquitecturas. Sin embargo, la cantidad de literatura sobre entornos inteligentes que utilizan ontologías y reglas de producción como bases representativas no es demasiado extensa. A continuación, se presenta una visión general de algunas de las técnicas de aprendizaje automático que se han utilizado para el aprendizaje de patrones humanos y que de alguna forma han influ ido en el trabajo que aquí se expone.

\subsection{Redes Ne uronales}

Entre los primeros grupos de investigación que se encargaron de desarrollar aplicaciones para entornos inteligentes donde los patrones de los usuarios estaban involucrados se encuentran los de Mozer et al.[3] y Chan et al.[4]. Por un lado, en[3] se encargaron de diseñar un sistema de control adaptativo del entorno llamado Neural Network House, que estudiaba el estilo de vida de sus habitantes y el consumo de energía. Para ello utilizaron una red neuronal para predecir dónde se iba a encontrar el usuario en los próximos segundos, y usaban esta información para controlar la ilu minación.

Por otra parte, Chan et a1.[4] desarrollaron un sistema que predecía la presencia o ausencia de usuarios, así como su localización. El sistema comparaba la ubicación actual con la predicción elaborada para determinar si la situación actual era normal o inusual.

Basada en una red neuronal auto-adaptativa conocida como GSOM (Growing Self-Organizing Maps), Zheng et al.[5] diseñaron una plataforma de minería de datos para el análisis de actividades humanas dentro de un entorno domótico. Además, esta propuesta proporciona varios métodos que permiten un mejor análisis en áreas de interés muy concretas.

\subsection{Aprendizaje Por Refuerzo}

Algunos de los grupos que hemos citado anteriormente han desarrollado un módulo basado en aprendizaje por refuerzo para dotar al entorno de la capacidad de adaptación.

En este sentido, Mozer et a1.[6] utilizaron Q-Learning para regular el alumbrado del entorno. El sistema trataba de minimizar el consumo de energía en tanto que el usuario no mostrase disconformidad, to mando como punto de partida la suposición de que el usuario no tenía preferencias iniciales.

\subsection{TÉCnicas de Clasificación}

Otros grupos de investigación han optado por utilizar técnicas de clasificación como árboles de decisión o reglas de inducción. Los investigadores que trabajan en el entorno conocido como SmartOffice[7] desarrollaron, utilizando árboles de decisión, una aplicación que generaba reglas para separar y distinguir situaciones donde los datos del entorno indicaban reacciones diferentes por parte del usuario.

Lühr et a1.[8] presentaron un trabajo de minería de datos basado en la aplicación de IAR (Intertransaction Association Rule) para la detección de comportamiento anómalo en los usuarios de una vivienda domótica. Las IARs son reglas de implicación que permiten capturar las relaciones, no secuenciales, de los eventos capturados dentro de una vivienda. En este proyecto se utilizaban sus propiedades para detectar el co mportamiento nuevo y, posiblemente anormal, en los habitantes y tomar partido de manera apropiada, co mo por ejemplo, consultar al usuario sobre el nuevo comportamiento, refrescarle la memoria acerca de una tarea que estaba haciendo o incluso alertar a algún familiar cuando sea necesario. Se trata, por tanto, de una propuesta orientada principalmente a las personas ancianas que viven de forma autónoma en sus viviendas.

\subsection{Descubrimiento de Secuencias}

El proyecto MavHome[9] presentaba una aplicación orientada a la construcción de modelos universales, representados mediante Modelos de Markov, para deducir las localizaciones o actividades futuras de los habitantes de un entorno inteligente. La información suministrada se utilizaba luego para automatizar los dispositivos y adaptarlos en el tiempo en base al algoritmo de decisión ProPHeT[10].

CASAS[11] es un proyecto realizado por el mis mo grupo que el anterior, el cual implementaba un modelo capaz de adaptarse a los individuos de un entorno domótico, descubriendo sus patrones frecuentes. Entre las aportaciones más recientes de este proyecto, se encuentra un método no supervisado de reconocimiento de las actividades que se utilizan para medir la salud funcional de los usuarios[12], de forma que pueda comprobarse si mantienen su rutina o no.

Por último, Aztiria et al.[13] propusieron un proceso de descubrimiento de acciones del usuario en un sistema basado en reconocimiento de voz. Este sistema permitía al individuo interactuar con los patrones para así usar su aprobación a la hora de automatizar las acciones.

\subsection{Conclusiones Sobre el Trabajo Relacionado}

Después de analizar todas estas aplicaciones, parece claro que el uso de una técnica u otra está condicionado por las necesidades concretas de cada entorno, tal y co mo apuntaba Muller[14]. Además, estos trabajos muestran que aún no existe una propuesta global de aprendizaje. En ese sentido, dadas las fortalezas y debilidades de distintas técnicas, la 
combinación de las mismas parece una estrategia prometedora. De este modo, nuestro módulo de aprendizaje se basa en algunas de las técnicas anteriores, especialmente en las relativas al descubrimiento de secuencias y en los conceptos ya expuestos en un trabajo previo[2], pero introduciendo nuevos parámetros configurables para crear un sistema donde el usuario pueda guiar al entorno a actuar de una manera personalizada, al mis mo tiempo que se utiliza la información adquirida para servir de apoyo en la to ma de las decisiones más importantes.

\section{Arquitectura del Modelo de Aprendizaje}

La capa de aprendizaje que se presenta en este artículo se enmarca dentro del proyecto IntelliDomo (http://www.intellidomo.es)[2], un sistema inteligente capaz de controlar automáticamente los dispositivos de una instalación domótica y en tiempo real mediante reglas SWRL. Su característica principal radica en la capacidad de razonamiento y de respuesta en base a los continuos cambios que se producen en una instalación domótica.

Cada evento capturado por los sensores y actuadores que están distribuidos por el entorno queda registrado. Para poder gestionar todo este conocimiento, IntelliDo mo está construido sobre una ontología denominada Domo OWL, cuyos conceptos están relacionados con los dispositivos del sis tema y que están basados parcialmente en los expuestos en la ontología DogOnt[15]. Esta ontología, Domo OW L, ha sido modelada para estar en sincronía con los dispositivos físicos que constituyen el entorno domótico, de forma que puedan almacenarse sus valores y propiedades destacadas, tal y como se aprecia en la Fig. 1. De este modo, los componentes domóticos podrán clasificarse según sean sensores o actuadores, y dentro de este último, en electrodomésticos, dispositivos del sistema eléctrico, dispositivos del sistema de calefacción y ventilación, etc. Además, las clases de dispositivos se relacionan con las de tipos de datos, de manera que a partir de la clase de dispositivo a la que pertenezca un componente físico concreto, pueda establecerse el tipo de datos que identifique su valor: continuo (como en el caso de sensores de temperatura, de luz, etc), discreto,... El bus EIB/KNX y la red X10 son los protocolos de comunicaciones soportados para nuestra instalación.

Además, la ontología trabaja junto a un conjunto de reglas SWRL preestablecidas. El usuario puede modificar dichas reglas o crear otras nuevas mediante la herramienta IntelliDomoRules y automatizar de esta forma el comportamiento del sistema. El motor de inferencias de esta herramienta, Jess[16], decide qué regla debe dispararse en cada instante conforme a las prioridades y preferencias establecidas. Dicho motor de inferencia nos va a permitir inferir nuevo conocimiento en base a los hechos representados en las reglas SWRL.

También existe una base de datos domótica (QDS_DB) donde IntelliDomo actualiza los valores de estado de los dispositivos físicos en tiempo real. De este modo, IntelliDomo se encarga de transferir los cambios instantáneamente desde el nivel físico (dispositivos) al lógico (base de datos) y viceversa, manteniendo la integridad de los datos.

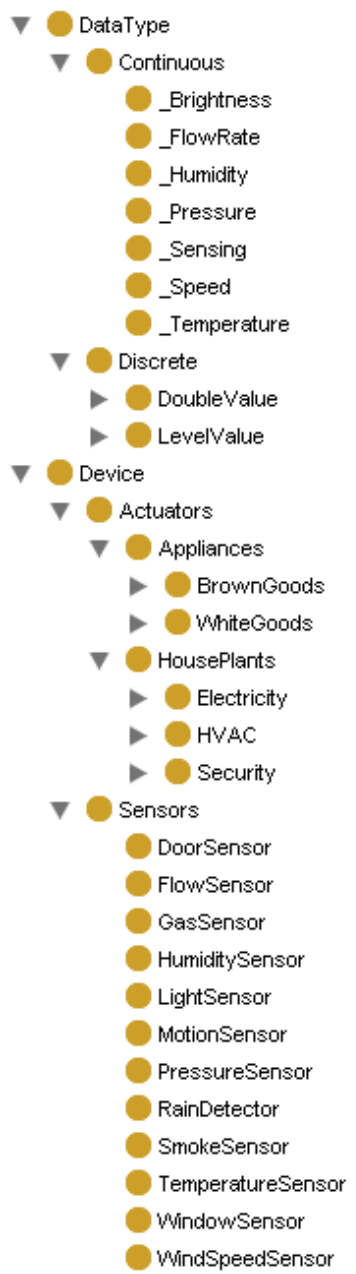

Figura 1. Clasificación general de la ontología Domo OWL

Finalmente, el módulo de aprendizaje que nos ocupa incorpora algoritmos para adquirir los hábitos de los usuarios, tanto los periódicos como los encadenados, y producir automáticamente reglas que contribuyan a tomar decisiones que satisfagan sus necesidades y anticipen sus hábitos más frecuentes. Por otra parte, los parámetros que rigen la ejecución del módulo de aprendizaje pueden ser configurados y personalizados por los propios usuarios si lo consideran necesario. De este modo, el sistema puede adaptarse a los cambios basándose en la retroalimentación implícita y exp lícita de los usuarios.

La arquitectura de dicha capa se presenta en la Fig. 2. Como puede observarse, el módulo de aprendizaje utiliza la información almacenada en la base de datos QDS_DB sobre los dispositivos del entorno y los eventos registrados para iniciar la búsqueda de patrones de comportamiento. En una primera etapa se descubren las actividades que el usuario repite con una cierta periodicidad para, posteriormente, 
detectar aquellas secuencias de acciones que se producen con frecuencia dentro de su vida diaria. Una vez se obtiene este conocimiento, se traduce en reglas de producción que el sistema pueda interpretar y lanzar mediante la herramienta IntelliDomoRules. A continuación se describe en profundidad cómo se lleva a cabo internamente este proceso de aprendizaje.

\section{Aprendizaje Para la Toma de Decisiones}

El aprendizaje de patrones de comportamiento no es un aspecto más del sistema que puede conllevar algunas ventajas para un entorno inteligente, sino que más bien lo consideramos como una aportación es encial para formalizar la idea de que un entorno pueda actuar inteligentemente. Proporciona respaldo a entornos que se adaptan a sus usuarios de forma discreta y de manera que dichos usuarios queden liberados de la carga de programar continuamente los dispositivos domóticos. Por tanto, la habilidad para aprender hábitos de comportamiento y actuar en consecuencia es de suma importancia para implementar con éxito un entorno inteligente.

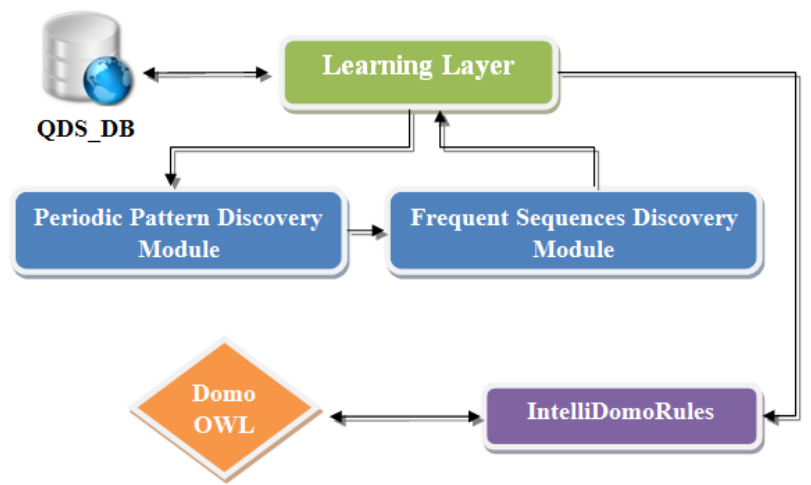

Figura 2. Arquitectura de la capa de aprendizaje de IntelliDomo

Los datos recolectados en un entorno inteligente están formados por las lecturas de todos los tipos de sensores y por la manipulación manual de los dispositivos por parte de los habitantes (luces, persianas, etc). Cada evento del conjunto de datos se considera una acción; por ejemplo, encender una luz del pasillo, poner el aire acondicionado a 20 grados... De aquí en adelante, asumiremos que los datos de entrada son una secuencia de tuplas definidas de la forma $\left\{\mathrm{d}_{\mathrm{i}}, \mathrm{s}_{\mathrm{i}}, \mathrm{t}_{\mathrm{i}}\right\}$, donde $\mathrm{d}_{\mathrm{i}}$ denota el dispositivo afectado, $\mathrm{s}_{\mathrm{i}}$ el estado de dicho dispositivo y $t_{i}$ la fecha y hora del evento. Por simplicidad, el sistema considera varios usuarios como uno sólo en el entorno.

El módulo de aprendizaje abarca las siguientes tareas sobre el conjunto de datos: identificar patrones periódicos en el tiempo, encontrar secuencias frecuentes de acciones encadenadas, y automatizar los patrones encontrados mediante la generación de reglas SWRL que permitan al sistema tomar decisiones conforme a los gustos y hábitos de los usuarios. En cuanto a la tarea de descubrimiento de patrones periódicos, ya se introdujo un algoritmo de aprendizaje de reglas en[2], basado en momentos del día, para describir las acciones periódicas de los usuarios y automatizarlas. Sin embargo, necesitaba de la revisión de los parámetros que rigen el funcionamiento del algoritmo, así como la incorporación de un nuevo módulo que analizase las relaciones entre acciones consecutivas, ya que nuestro objetivo es establecer las bases para el desarrollo de una propuesta global que mejore la adaptación del entorno hacia sus usuarios y les quite carga de trabajo, y que pueda reutilizarse y extenderse gracias al uso de ontologías y reglas de producción.

La manera de completar esta propuesta de aprendizaje implica analizar las actividades desarrolladas por los habitantes y descubrir no sólo patrones periódicos, sino también secuencias comunes de acciones consecutivas. En definitiva, este módulo de aprendizaje debe enfocarse en la información de contexto y el razonamiento temporal.

A continuación se describen cada una de las fases en que se divide el proceso de aprendizaje que se ha desarrollado.

\subsection{Primera Fase: Descubrir Patrones Periódicos}

Como ya se ha mencionado, gran parte del desarrollo de esta fase está incluida en los trabajos previos [2] y[17], por lo que en este sub-apartado se presenta un resumen de la mis ma para entender los nuevos cambios añadidos a partir de la siguiente etapa. Las bas es delalgorit mo que rige este módulo están fuertemente influenciadas por el algoritmo Apriori de Rekesh Agrawal[18]. El objetivo principal de esta primera fase consiste en detectar acciones periódicas, basadas en el razonamiento temporal, a part ir de los datos de entrada. Un patrón se describe como un evento periódico, bien definido y aislado. Estas tres características se explican a continuación.

Consideremos la frecuencia $f_{i}$ como el número de veces que una determina acción $\left\{\mathrm{d}_{\mathrm{i}}, \mathrm{s}_{\mathrm{i}}, \mathrm{t}_{\mathrm{i}}\right\}$ se repite en el conjunto de datos. Para poder calcularla, se debe definir un rango de acción $r_{i}=\left[t_{i}-\alpha, t_{i}+\alpha\right]$, donde $\alpha$ es una constante para ampliar o reducir el radio de búsqueda, para establecer el intervalo de tiempo a considerar en las repeticiones, ya que las acciones diarias de los usuarios no se repiten exactamente a la mis ma hora. De este modo, otra tupla $\left\{\mathrm{d}_{\mathrm{j}}, \mathrm{s}_{\mathrm{j}}, \mathrm{t}_{\mathrm{j}}\right\}$ se considerará una repetición de la anterior si afecta al mis mo dispositivo de la mis ma forma $\left(\mathrm{d}_{\mathrm{i}}=\mathrm{d}_{\mathrm{j}} \wedge \mathrm{s}_{\mathrm{i}}=\mathrm{s}_{\mathrm{j}}\right)$ y su marca de tiempo está dentro del rango de acción ( $\left.\mathrm{t}_{\mathrm{j}} \in\left[\mathrm{t}_{\mathrm{i}}-\alpha, \mathrm{t}_{\mathrm{i}}+\alpha\right]\right)$.

Una frecuencia de acción se considerará relevante cuando alcance un determinado umbral $\mu$ al que lla maremos soporte mínimo, y que viene dado por el tamaño del conjunto de datos de entrada. Un patrón se considerará periódico y bien definido cuando se repita frecuentemente alcanzando el soporte mínimo y con una cierta periodicidad $\left(f_{i}>=\mu\right)$.

La periodicidad representa la regularidad de ocurrencia de las acciones o actividades. El módulo de aprendizaje considera varios tipos de periodicidad: diaria, semanal, mensual,... ya que existen comportamientos diferentes dependiendo de estos tipos de temporalidad.

Debido a la naturaleza errática de los humanos, diferentes 
tipos de actividades pueden mezclarse en el tiempo, generando ruido en los datos y provocando dificu ltades en la detección de patrones periódicos. Por esta razón, el algoritmo establece un nuevo valor umbral $\eta$ para eliminar dicho ruido y determinar si un conjunto de acciones puede identificarse claramente como una única unidad o no. Este parámetro se denomina porcentaje de ruido.

Un patrón periódico y bien definido se considera aislado cuando no excede el porcentaje de ruido en los datos.

El análisis de secuencias de eventos encadenados, principal aportación de este artículo, se estudia en un nuevo proceso de minería de datos, basado en el anterior, que veremos en el siguiente sub-apartado.

\subsection{Segunda Fase: Descubrir Secuencias de Acciones Frecuentes}

La segunda fase tiene como objetivo descubrir secuencias frecuentes de acciones consecutivas, y está influenciada por el algoritmo ED (Episode Discovery)[10]. En este caso, la marca de tiempo de dichas acciones ya no goza de tanta importancia como en el módulo anterior. Lo importante ahora es detectar que cada vez que una acción " $\mathrm{A}$ " tiene lugar, una secuencia de acciones "B", “C", ... tendrá lugar después, tal y como se aprecia en la Fig. 3. En cuanto al descubrimiento de secuencias de acciones o actividades, no tiene sentido analizar exclusivamente el razonamiento temporal, puesto que en ese caso la frecuencia que determina si una acción puede considerarse como un hábito frecuente viene dada por repeticiones cercanas en el tiempo.

\section{A B CD F H E A C C E B H F A B C}

Figura 3. Ejemplo de patrón frecuente $\mathrm{ABC}$

En este sentido, para estudiar las relaciones de acción-acción se necesita la evaluación de otros aspectos para poder descubrir secuencias de acciones adecuadamente a partir del conjunto de datos. En otras palabras, si una acción no se repite periódicamente a una hora concreta sino que lo hace de forma dispersa, no significa necesariamente que el sistema deba ignorarla, ya que su aparición en el conjunto de datos puede deberse a un patrón de secuenciación con otras acciones.

Veámoslo con un ejemplo: supongamos que se observa en un conjunto de datos dado que el usuario enciende todos los días el calentador del agua para darse una ducha. No obstante, resulta difícil establecer un patrón de comportamiento que se anticipe a esta acción ya que el usuario la lleva a cabo en momentos muy distantes. El proceso de aprendizaje basado en el razonamiento temporal habría descartado esta información. Sin embargo, un análisis más profundo del contexto nos lleva a descubrir que cada vez que el usuario enciende el calentador, a los 2 minutos enciende también la cafetera y el tostador para calentar el café y las tostadas del desayuno mientras toma la ducha. Como consecuencia, ahora es posible generar un patrón de comportamiento para la actividad llamada "preparar el desayuno", de forma que cada vez que se active el calentador del agua por la mañana, se dispare una regla de producción que encienda a los 2 minutos tanto la cafetera co mo la tostadora.

Desde ahora, para el caso de aprendizaje de secuencias de acciones asumiremos que una frecuencia fi es relevante cuando una secuencia de dos o más acciones se repita en el conjunto de datos de partida, lo que denominaremos actividad. Por tanto, una actividad x vendrá definida de la forma $x=(\{\mathrm{di}, \mathrm{si}, \mathrm{ti}\},\{\mathrm{dj}, \mathrm{sj}, \mathrm{tj}\}, \ldots)$, donde la primera acción del conjunto será quien desencadene el resto de acciones que conforman la actividad.

Llamaremos tamaño de ventana a las dimensiones del marco de búsqueda de actividades en un instante dado, de forma que un tamaño de ventana igual a 2 implicará buscar actividades que involucren a dos elementos domóticos, un tamaño de ventana 3 será de búsqueda de actividades que encadenen 3 acciones, etc. Por tanto, un tamaño de ventana w (inicializado a 1) se pasará sobre el conjunto de datos, de modo que en cada instante se proces arán aquellas secuencias cuya longitud sea igual al tamaño de ventana dado. A continuación se detallan los pasos a seguir en función del tamaño de ventana.

Primer paso - Tamaño de ventana igual a 1: En este paso, lo que se pretende es limpiar el conjunto de datos de entrada y prepararlo con aquellas acciones más habituales. Una acción se considerará frecuente si su número de repeticiones supera el soporte mínimo establecido, es decir, si f(\{di, si, ti $\})>=\mu$, donde $\mathrm{f}$ es el número de veces que el par $\{\mathrm{di}, \mathrm{si}\}$ aparece en el conjunto de datos. Como vemos, ya no es necesario buscar acciones dentro de un intervalo de tiempo concreto. De es ta forma, se evita que el algorit mo to me co mo ciertas determinadas secuencias de acciones poco contrastadas que puedan ser fruto de una situación circunstancial.

Segundo paso - Tamaño de ventana mayor o igual a 2: Este paso constituye el proceso principal del módulo de aprendizaje de secuencias de acciones. En pocas palabras, se trata de comprobar para cada una de las acciones que se reciben del paso anterior si forman parte de una secuencia frecuente de acciones encadenadas. Por tanto, el procesamiento de dichas acciones consistirá en un bucle en el que el candidato se va ampliando paulatinamente conforme al tamaño de ventana, hasta establecer la longitud máxima de la actividad x en cuestión. En primer lugar, se parte del conjunto de acciones frecuentes del paso anterior $y$, para cada una de ellas, se analiza su relación con el resto, aumentando paulatinamente el tamaño de ventana y enlazándola secuencialmente con tantas como sea posible. La periodicidad de las acciones encadenadas debe ser la mis ma. Para comprobar si dos o más acciones están relacionadas de manera relativa en el tiempo, se contabiliza el número de veces que dada la primera acción que in icia la actividad se produce a continuación la segunda acción, y luego la tercera... y así sucesivamente. Si se producen en un porcentaje de veces significat ivo y el espacio de tiempo entre las acciones es generalmente el mismo, entonces podrá decirse que constituyen una actividad o secuencia de acciones habitual. Para este paso, el soporte mínimo nos 
indicará si el porcentaje de ocurrencias de la actividad en conjunto es importante o no, mientras que el rango de acción nos ayudará a comprobar si la distancia de tiempo entre las acciones suele estar siempre oscilando en el mismo intervalo. Cuando se ha terminado de procesar una acción para ver si forma parte de una actividad, se to ma la siguiente dentro de los datos de entrada y se repite nuevamente el proceso hasta haber analizado todas las acciones. En caso de formarse una actividad, se tomará la primera acción $\{\mathrm{di}, \mathrm{si}, \mathrm{ti}\}$ como integrante del contexto que activará la ejecución de dicha actividad, anotándose el rango de tiempo $\mathrm{t}=[\mathrm{ti}+\alpha, \mathrm{ti}-\alpha]$. en que se produce.

\subsection{Tercera Fase: Validando el Proceso de Aprendizaje}

El usuario puede rellenar los parámetros de configuración descritos arriba, así como decidir qué eventos del conjunto total de datos van a ser evaluados por el módulo. Sin embargo, por lo general el proceso de aprendizaje se ejecutará offline en intervalos regulares confirmados por el usuario.

En cuanto finaliza el proceso de aprendizaje, el sistema genera automáticamente una regla SWRL para cada patrón y el usuario puede decidir cuáles de ellas se van a activar para ser procesadas por IntelliDomo Rules. La transformación de estos patrones en reglas se realiza a través del parser de IntelliDo mo, que se encarga de convertir las cadenas de texto generadas en la capa de aprendizaje en SW RLAtoms. Estos átomos tienen un formato predefinido, que es el siguiente: Propiedad (Instancia, Valor) y Clase (Instancia). Además, existe otro tipo especial de átomos, denominados Built-inAtoms, que permiten al usuario definir otros formatos de predicado y su interpretación. La Fig. 4 muestra un ejemplo de una actividad transformada ya en formato SWRL y de cómo son dichos átomos.

Condition: value(MotionSens or, 1 )^ equals HourAndMinute("08:00","08:30")

Action: $\rightarrow$ value(Radio, 1)^value(AirConditioning, 22.00)

Figura 4. Ejemplo de actividad en formato de regla SWRL

Por último, las reglas aceptadas se unen a las ya existentes en una escala de prioridad, que puede cambiarse más adelante. Cada regla SW RL se almacena tanto en la base de datos como en la ontología. Las reglas almacenadas en la base de datos, se guardan junto con su marca de tiempo. Esta marca temporal, se crea en base al evento al que la regla va a anticiparse. De esta forma, por ejemplo, si IntelliDomo ha detectado que un evento sucede entre las 8:00 am y las 8:05 am, la regla se generará para que sea disparada unos minutos antes de las 8:00 am. El módulo encargado de ejecutar el motor de inferencia de IntelliDomo (Jess), sólo disparará aquellas reglas que estén dentro de ese rango temporal.

Cuando una regla es disparada, puede que la acción que realiza esa regla no se adapte a las necesidades que tiene el usuario en ese momento. Por tanto, si se ejecuta una regla y el usuario lleva a cabo la acción contraria repetidas veces, esa regla será eliminada del conjunto de reglas, ya que se ha detectado que el usuario no quería que ese evento tuviera lugar en ese momento. Esto permite al sistema aprender de nuevo cuándo debe ejecutar esa regla y cuándo no.

\section{Caso de Estudio}

En esta sección se describe uno de los casos de prueba que hemos desarrollado con el fin de comprender mejor los conceptos mencionados anteriormente y entender su aplicabilidad en casos como la asistencia médica.

En primer lugar, se considera el siguiente escenario para llevar a cabo el experimento: Antonio es un anciano de 60 años que vive sólo y al que le gustaría disponer de un sistema que le facilitase su vida diaria. Los días laborables se levanta poco después de que suene la alarma a las 08:00 a.m. y luego se viste. Sin embargo, los lunes, miércoles y viernes suele ir al baño unos 10 o 20 minutos después de despertarse, encendiendo previamente el calentador. Es entonces cuando aprovecha para darse una ducha, poniendo la temperatura del agua en 24 grados. Después de salir del baño, sube todas las persianas. Cuando entra en la cocina, enciende la telev isión y pone en marcha la cafetera para desayunar mientras ve su programa favorito. A las 09:00 a.m., tras haber desayunado, se marcha de la casa. A las 03:00 p.m. regresa de trabajar y enciende la calefacción para mantener el ambiente acondicionado lo que resta del día. A continuación, unos 30 minutos después vuelve a la cocina para calentarse la comida y comer. Cuando termina, se retira a su habitación para descansar. Los miércoles, cuando se levanta de la siesta, se dirige al salón para encender la radio y escuchar el programa deportivo mientras lee el periódico junto a la luz de un flexo. Todos los días suele cenar en el salón para irse posteriormente a la cama sobre las 11:00 p.m.

El escenario anterior es propicio para automatizar los hábitos del individuo y quitarle carga de trabajo, al mis mo tiempo que la información adquirida puede ser utilizada por personal médico para corregir malos hábitos y detectar posibles casos de Alzheimer (por ejemplo, si se detectan signos de desorientación al observarse que el individuo vuelve con frecuencia a sitios de la casa donde ya ha estado).

Para llevar a cabo nuestro proceso de aprendizaje, todas las acciones que lleva a cabo el usuario sobre los dispositivos ubicados en este escenario, incluida la información capturada por los distintos tipos de sensores, quedan registradas en la base de datos de IntelliDomo. Para la recogida de estos datos, se ha establecido un período de estudio de 4 meses, en el que se ha registrado toda la información que formará parte del conjunto de datos para la evaluación.

Una vez que la base de datos de log está preparada con información suficiente, el individuo tiene la opción de configurar los parámetros del módulo de aprendizaje. En este caso, se ha optado por dejar los valores por defecto: soporte mínimo de un $60 \%, 5$ minutos de rango de acción, porcentaje de ruido del $30 \%$, y periodicidad diaria y semanal. En 
general, el módulo de aprendizaje se ejecutará offline en intervalos regulares (una vezal mes).

Para este escenario de prueba, se ha analizado el conjunto de datos con dos versiones del aprendizaje, la del trabajo previo (sólo patrones periódicos) y la que se presenta en este artículo (patrones periódicos y secuencias de acciones). Los patrones de comportamiento generados por cada una de las versiones se muestran en la Tabla 1 y la Tabla 2, respectivamente.

Tabla 1. Resultados de la versión anterior

\begin{tabular}{|c|c|c|c|}
\hline Fuente & Estado & Periodicidad & Contexto \\
\hline Alarm 1 & On & Daily & $08: 00: 02$ \\
\hline KitchenTV & On & Tuesday & $08: 16: 21$ \\
\hline All lights & Off & Daily & $09: 00: 16$ \\
\hline Heating1 & On & Daily & $15: 01: 03$ \\
\hline Cooker & On & Daily & $15: 30: 24$ \\
\hline Heating1 & Off & Wednesday & $22: 52: 14$ \\
\hline All lights & Off & Daily & $23: 00: 05$ \\
\hline
\end{tabular}

Tabla 2. Resultados de la versión actual

\begin{tabular}{|c|c|c|c|}
\hline Fuente & $\begin{array}{c}\text { Estad } \\
0\end{array}$ & Periodicidad & Contexto \\
\hline Alarm 1 & On & Daily & 08:00:02 \\
\hline $\begin{array}{c}\text { Heater } \\
\text { WaterTemp } \\
\text { All blinds } \\
\end{array}$ & $\begin{array}{l}\text { On } \\
24 \\
\text { Up } \\
\end{array}$ & Monday & $\begin{array}{c}\text { BedroomSensor } \\
\text { Off[08:00:00 - } \\
08: 30: 00]\end{array}$ \\
\hline $\begin{array}{c}\text { Heater } \\
\text { WaterTemp } \\
\text { All blinds }\end{array}$ & $\begin{array}{l}\text { On } \\
24 \\
\text { Up }\end{array}$ & Wednesday & $\begin{array}{c}\text { BedroomSensor } \\
\text { Off[08:00:00 - } \\
08: 30: 00]\end{array}$ \\
\hline $\begin{array}{c}\text { Heater } \\
\text { Wat erTemp } \\
\text { All blinds } \\
\end{array}$ & $\begin{array}{l}\text { On } \\
24 \\
\text { Up } \\
\end{array}$ & Friday & $\begin{array}{c}\text { BedroomSensor } \\
\text { Off[08:00:00 - } \\
08: 30: 00] \\
\end{array}$ \\
\hline $\begin{array}{c}\text { KitchenT V } \\
\text { CoffeeMaker } \\
\text { CoffeeMaker }\end{array}$ & $\begin{array}{l}\text { On } \\
\text { On } \\
\text { Off } \\
\end{array}$ & Daily & $\begin{array}{c}\text { KitchenLight } \\
\text { On[08:38:00 - } \\
09: 00: 00] \\
\end{array}$ \\
\hline All lights & Off & Daily & 09:00:16 \\
\hline Heat ing1 & On & Daily & $15: 01: 03$ \\
\hline Cooker & On & Daily & $15: 30: 24$ \\
\hline $\begin{array}{l}\text { BedroomBli } \\
\text { nd }\end{array}$ & Down & Daily & $\begin{array}{c}\text { BedroomSensor } \\
\text { On[16:10:00 - } \\
16: 20: 00] \\
\end{array}$ \\
\hline $\begin{array}{c}\text { Radio } \\
\text { ReadingLam } \\
\mathrm{p} \\
\end{array}$ & $\begin{array}{l}\text { On } \\
\text { On }\end{array}$ & Wednesday & $\begin{array}{c}\text { LoungeSensor } \\
\text { On[17:15:00- } \\
17: 47: 00] \\
\end{array}$ \\
\hline All lights & Off & Daily & $23: 00: 05$ \\
\hline
\end{tabular}

A raíz de los resultados, el usuario tiene ahora la opción de validarlos y decidir qué patrones se van a automatizar (las filas sombreadas en gris son las que el usuario ha rechazado). Para la valoración de los resultados obtenidos, se ha analizado tanto el número de falsos positivos (reglas no validadas por el usuario) co mo el de falsos negativos (reglas que existen pero que no se han adquirido). En el mejor de los casos se esperaba adquirir 11 patrones, los que obtiene correctamente la segunda versión más otro relacionado con la actividad "cenar".

Como puede observarse en la Tabla 3, donde se muestra la valoración de los resultados, la nueva versión del proceso de aprendizaje mejora significativamente los resultados de la versión anterior para este caso de estudio, gracias al análisis de secuencias de acciones frecuentes en función del contexto. Sin embargo, aún puede mejorarse el porcentaje de falsos positivos y falsos negativos para conseguir que el entorno se adapte lo mejor posible a los individuos.

Tabla 3. Valoración de los resultados

\begin{tabular}{|c|c|c|c|}
\hline \multirow{2}{*}{ Versión } & \multicolumn{3}{|c|}{ Resultados obtenidos (en porcentajes) } \\
\cline { 2 - 4 } & Aciertos & Falsos posit ivos & Falsos negat ivos \\
\hline Anterior & $71,43 \%(5$ de 7$)$ & $28,57 \%(2$ de 7$)$ & $54,54 \%(6$ de 11$)$ \\
\hline Actual & $90,91 \%(10$ de 11$)$ & $9,09 \%(1$ de 11$)$ & $9,09 \%(1$ de 11$)$ \\
\hline
\end{tabular}

En general, para el número de experimentos realizados hasta ahora (50 ejecuciones por cada uno de los 7 escenarios probados ), la media de falsos positivos es del orden del $15 \%$, mientras que la de falsos negativos es del orden del $20 \%$.

\section{Conclusiones y Trabajo Futuro}

La Inteligencia Ambiental es una de las áreas de investigación actuales que está ganando importancia en el desarrollo de entornos centrados en los usuarios. Con este trabajo se persigue descubrir las actividades periódicas y frecuentes de los humanos dentro de un entorno domótico, ya que al automatizarlas se consigue que el entorno sea receptivo hacia sus usuarios, quitándoles la carga de trabajo de las tareas repetitivas y favoreciendo la monitorización de dichas actividades, lo cual puede resultar útil en el ámbito sanitario. Hasta ahora, en los trabajos anteriores nos habíamos centrado en automatizar exclusivamente las acciones periódicas en el tiempo, sin considerar las relaciones entre los distintos eventos capturados a lo largo del día. Finalmente, con la incorporación de un nuevo módulo de aprendizaje para el descubrimiento de secuencias de acciones comunes, se ha conseguido completar y mejorar el trabajo previo como así indican las pruebas realizadas hasta el momento.

Por otro lado, la combinación del análisis de contexto con el razonamiento temporal representa una técnica prometedora para comprender el comporta miento humano y permitir al entorno actuar y decidir conforme a las preferencias y prioridades de sus usuarios. La incorporación de parámetros configurables junto con el uso de ontologías y reglas de producción SWRL se ha realizado buscando la adaptación del modelo hacia una propuesta global que sea fácil de usar por los usuarios y cuyo conocimiento sea reutilizable.

Actualmente estamos desarrollando módulos específicos de toma de decisiones para adaptar esta propuesta a escenarios concretos y más sustanciosos (eficiencia energética, asistencia sanitaria, seguridad,...), ya que si el 
entorno conoce cómo se comportan los usuarios, puede tomar decisiones que automaticen esos hábitos mientras mejoran su funcionamiento en base a diferentes aspectos.

\section{AGRADECIMIENTOS}

Este trabajo se ha desarrollado con el apoyo y soporte de Cátedra Telefónica de la Universidad de Extremadura, FEDER, TIN 2011-27340 y Junta de Extremadura.

\section{REFERENCIAS}

[1] V. Rialle, C. Ollivet, C. Guigui and C. Hervé, "What do family caregivers of alzheimer's disease patients desire in smart home technologies? Contrasted results of a wide survey," in Methods of Information in Medicine, vol. 47, pp. 63-69, 2008.

[2] P. Valiente-Rocha, J. L. Redondo-García and A. Lozano-Tello, "Ambient intelligence system for controlling home automation instalations," Fifth Iberian Conference on Information Systems and Technologies, pp. 1-6, June 2010.

[3] M. C. Mozer, R. H. Dodier, M. Anderson, L. Vidmar, R. F. Cruickshank and D. Miller, "The neural network house: an overview," in Current trends in connectionism, L. Niklasson and M. Boden, Eds.Erlbaum, pp. 371-380, 1995.

[4] M. Chan, C. Hariton, P. Ringeard and E. Campo, "Smart house automation system for the elderly and the disabled," IEEE International Conference on Systems, Man and Cybernetics, pp. 1586-1589, 1995.

[5] H. Zheng, H. Wang and N. Black, "Human activity detection in smart home environment with self-adaptive neural networks," IEEE International Conference on Networking, Sensing and Control, pp. 1505-1510, May 2008.

[6] M. C. Mozer, "Lessons from an adaptive home," in Smart Environments: Technology, Protocols and Applications, D. J. Cook and S. K. Das, Eds. Wiley-Interscience, pp. 273-298, 2004.

[7] C. L. Gal, J. Martin, A. Lux and J. L. Crowley, "Smartoffice: design of an intelligent environment," in IEEE Intelligent Systems, vol. 16, no 4, pp. 60-66, 2001
[8] S. Lühr, G. West and S. Venkatesh, "Recognition of emergent human behavior in a smart home: a data mining approach," in Pervasive Mobile Computing, vol. 3, issue 2, pp. 95-116, 2007.

[9] G. M. Youngblood, D. J. Cook and L. B. Holder, "M anaging adaptive versatile environments," in Pervasive and Mobile Computing, vol. 1, no 4, pp. 373-403, 2005.

[10] G. M. Youngblood and D. J. Cook, "Data mining for hierarchical model creation," in IEEE Transactions on Systems, Man and Cybernetics, Part C: Aplications and Reviews, vol. 37, no 4, pp. 561-572, July 2007.

[11] P. Rashidi and D. J. Cook, "Keeping the resident in the loop: adapting the smart home to the user," IEEE Transactions on Systems, Man and Cybernetics, Part A: Systems and Humans, vol. 39, no 5, pp. 949-959, 2009.

[12] P. Rashidi, D. Cook, L. Holder, and M. Schmitter-Edgecombe, "Discovering activities to recognize and track in a smart environment," IEEE Transactions on Knowledge and Data Engineering, vol. 23, no 4, pp. 527-539, 2011.

[13] A. Aztiria, A. Izaguirre, R. Basagoiti and J. C. Augusto, "Learning about preferences and common behaviours of the user in an intelligent environment," in Behaviour Monitoring and Interpretation -BMI- Smart Environments, Ambient Intelligence and Smart Environments book series, vol. 3, pp. 289-315, 2009.

[14] M. E. Muller, "Can user models be learned at all? inherent problems in machine learning for user modelling," in Knowledge Engineering Review, vol. 19, Cambridge University Press, pp. 61-88, 2004.

[15] D. Bonino and F. Corno, "DogOnt - Ontology modelling for intelligent domotic environments," Seventh International Semantic Web Conference, pp. 790-803, October 2008.

[16] E. J. Friedman-Hill, "Jess in action: rule-based systems in java," Manning Press, 2003.

[17] V. Botón-Fernández and A. Lozano-Tello, "Learning algorithm for human activity detection in smart environments," IEEE/WIC/ACM International Conference on Web Intelligence and Intelligent Agent Technology, pp. 45-48, August 2011.

[18] R. Agrawal and R. Srikant, "Mining sequential patterns," Eleventh International Conference on Data Engineering, pp. 3-14, March 1995. 